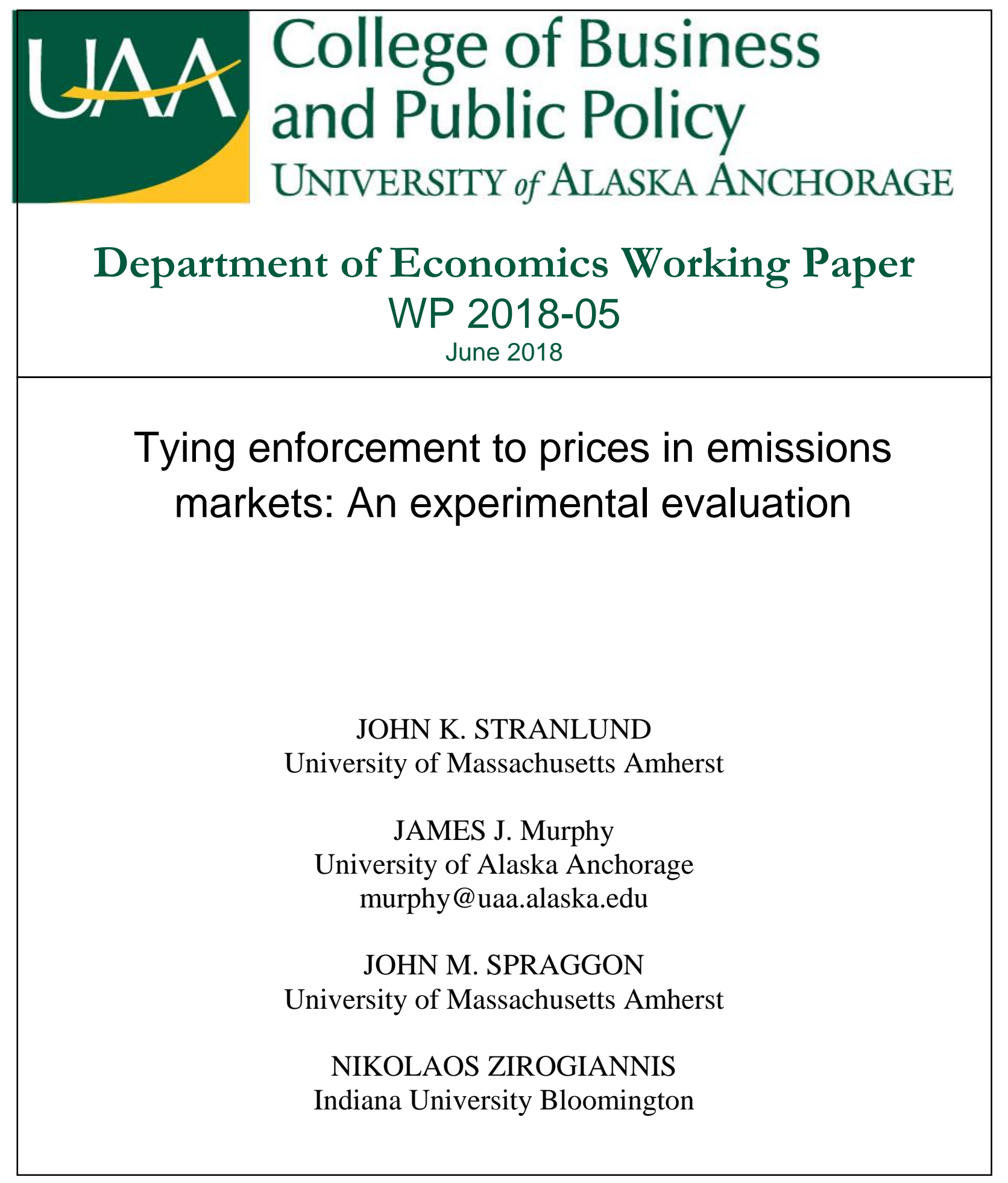

UAA DEPARTMENT OF ECONOMICS

3211 Providence Drive

Rasmuson Hall 302

Anchorage, AK 99508

http://econpapers.uaa.alaska.edu/ 


\section{Tying enforcement to prices in emissions markets: An experimental evaluation}

John K. Stranlund

Department of Resource Economics

University of Massachusetts-Amherst

James J. Murphy

Department of Economics

University of Alaska-Anchorage

John M. Spraggon

Department of Resource Economics

University of Massachusetts-Amherst

Nikolaos Zirogiannis

School of Public and Environmental Affairs

Indiana University-Bloomington

Acknowledgements: This research has been supported by a grant from the U.S. Environmental Protection Agency's National Center for Environmental Research (NCER) Science to Achieve Results (STAR) program. Although the research described in the article has been funded wholly or in part by the U.S. EPA through grant number RD-83367201-0, it has not been subjected to the EPA's peer and policy review and therefore does not necessarily reflect the views of the Agency, and no official endorsement should be inferred. Additional funding provided by the USDA/NIFA, Massachusetts Experiment Station Project No. MAS00453. 


\section{Tying enforcement to prices in emissions markets: An experimental evaluation}

Abstract: We present results from laboratory emissions permit markets designed to investigate the transmission of abatement cost risk to firms' compliance behavior and regulatory enforcement strategies. With a fixed expected marginal penalty, abatement cost shocks produced significant violations and emissions volatility as predicted. Tying the monitoring probability to average permit prices effectively eliminated noncompliance, but transmitted abatement cost risk to monitoring effort. Tying the penalty to average prices reduced violations, but did not eliminate them. Some individuals in these treatments sold permits at low prices, presumably in an attempt to weaken enforcement. While tying sanctions directly to prevailing permit prices has theoretical and practical advantages over tying monitoring to prices, our results suggest that this strategy may not be as effective as predicted without additional modifications.

Keywords: Emissions markets, risk and uncertainty, incomplete information, permit markets, compliance, enforcement, laboratory experiments.

JEL Codes: C91, L51, Q58

\section{Introduction}

Analysts and policymakers have long recognized that a regulator's uncertainty about emission control costs can affect decisions about when to implement emissions markets and how to design them (Weitzman 1974; Roberts and Spence 1976). In addition, emissions markets have to be enforced well if they are to achieve their goals. One of the most important lessons of the literature on enforcing emissions markets is that the competitive market price of emissions permits determines a firm's marginal benefit of violating their permits, and therefore is critical in determining compliance behavior and enforcement strategies (Malik 1990; Murphy and Stranlund 2006, 2007; Stranlund 2017). Thus, the risk associated with regulatory uncertainty about abatement costs can be transmitted to enforcement effort and compliance choices via changes in the price of emissions permits. Consequently, we have conducted a series of laboratory experiments to examine this transmission of abatement cost risk.

Our work is motivated by, and contributes to, two literatures. The first is the literature on policy choice under uncertainty. Weitzman's (1974) seminal work on prices versus quantities under uncertainty remains relevant to this day. Weitzman's results suggest that an emissions tax is the preferred instrument if the marginal damage function is not as steeply sloped as the marginal abatement cost function, while an emissions market with a fixed number of tradable permits is preferred if the relative steepness of the marginal damage and marginal abatement cost 
functions is reversed. An emissions tax allows the transmission of the abatement cost risk to emissions but not to the emissions price, while an emissions market fixes aggregate emissions so that abatement cost risk is only transmitted to the emissions price. Roberts and Spence (1976) constructed an emissions market with price controls that is often more efficient than a simple tax or a simple market because it spreads the transmission of abatement cost risk to both emissions and the emissions price in an efficient manner. More recent work on markets with price controls for greenhouse gases has continued to push this line of inquiry forward (e.g., Burtraw et al. 2010, Fell and Morgenstern 2010, Weber and Neuhoff 2010, Grull and Taschini 2011). Moreover, many proposed and existing emissions markets have some form of price control (Hood 2010, Newell et al. 2013).

The other literature that our work contributes to is the literature on enforcing emissions permit markets. See Stranlund (2017) for a thorough review of this literature. The fundamental problem of enforcing emissions markets is to make sure that pollution sources hold enough permits to cover their current emissions. This requires that regulators have systems in place to track emissions permits, but the much more difficult problem is to monitor sources' emissions. Moreover, both monitoring effort and applying sanctions are costly activities. Economists have examined the consequences of noncompliance in permit markets (Malik 1990, Stranlund and Dhanda 1999, Murphy and Stranlund 2006, 2007); compliance outcomes in the presence of market power (van Egteren and Weber 1996, Malik 2002, Chavez and Stranlund 2003); the effects of permit banking on compliance choices (Innes 2003, Stranlund et al. 2005; Cason and Gangadharan 2006; Stranlund et al. 2011); the efficient design of emissions markets, including their enforcement components (Stranlund 2007, Caffera and Chavez 2011), and other enforcement-related concerns.

While the literatures about policy choices under abatement cost uncertainty and enforcing emissions markets are both extensive, only a few authors have tied regulator uncertainty about abatement costs, the instrument choice problem, and enforcement of emissions markets together in theoretical models. The first to do so was Montero (2002), who reexamined the instrument choice problem under imperfect and costly enforcement. He found that an expected marginal penalty for permit violations that is lower than what would be necessary to induce full compliance under all realizations of aggregate abatement costs serves as a price ceiling as envisioned by Roberts and Spence (1976). If firms' abatement costs turn out to be high enough, 
then the permit price will rise to the expected marginal penalty and firms will increase their emissions beyond the permitted cap by violating their permits. Allowing the transmission of abatement cost risk to compliance choices can improve the performance of an emissions market.

However, Stranlund and Moffitt (2014) note several problems with using imperfect enforcement to provide a price ceiling in emissions markets. First, sanctioning noncompliant firms is not costless, so using imperfect enforcement to mitigate abatement cost risk involves the possibility of incurring costs to impose sanctions on noncompliant firms. Second, imperfect enforcement allows the transmission of abatement cost risk to compliance choices and to sanctioning actions and their costs. Finally, imperfect enforcement cannot be used to motivate extra emissions control when abatement costs turn out to be lower than expected. Stranlund and Moffitt (2014) propose a design that features enforcement to eliminate violations under all circumstances, an explicit price ceiling and a floor, and a sanction that varies directly with the permit price. This design addresses both low side and high-side abatement cost risk; full compliance eliminates variable sanctioning costs and emissions volatility, ${ }^{1}$ and making sanctions vary with the permit price prevents the transmission of abatement cost risk to monitoring efforts and its costs. Tying sanctions to permit prices is uncommon in actual emissions markets, but they were proposed for the US Clear Skies Initiative (US EPA 2003) and the US American Clean Energy and Security Act of 2009 (US Congress 2009), and were implemented in pilot programs of China's $\mathrm{CO}_{2}$ emissions trading system (Zhang 2015). ${ }^{2}$

Following the theoretical work of Montero (2002) and Stranlund and Moffitt (2014), in this paper we examine the transmission of abatement cost risk to compliance choices and enforcement actions with a series of laboratory experiments. These experiments consisted of markets with eight participants competing for a limited number of emissions permits (called production rights in the experiments), randomly changing abatement costs (called production benefits), and the possibility of imperfect compliance. In each round, subjects had to determine their emissions levels, trade for their desired number of permits, and consequently decide

\footnotetext{
${ }^{1}$ We define volatility as the change in average outcomes between periods.

2 The permit violation penalty in the U.S. Clear Skies Initiative was to be one to three times the clearing price in a recent permit auction. Similarly, the penalty in the American Clean Energy and Security Act of 2009 was set at twice the clearing price in a recent auction of $\mathrm{CO}_{2}$ permits. These initiatives were never enacted so their penalties were never implemented. Perhaps the only example of this form of sanction in actual use is in the pilot programs for China's $\mathrm{CO}_{2}$ emissions trading system. Most of these pilot programs set penalties at three to five times the average market price of $\mathrm{CO}_{2}$ allowances.
} 
whether to violate their permits and by how much. Our initial design consisted of three treatments. The Baseline treatment involved a fixed monitoring probability of 0.5 and a fixed sanction that produced a constant expected marginal penalty that was not high enough to motivate full compliance by risk-neutral firms, except when abatement costs were at their lowest level. We hypothesized that there would be significant violations in this treatment, and significant volatility in both emissions and violation choices. In practice, this transmission of risk to compliance choices results in the transmission of risk to sanctioning actions and their costs. On the other hand, in the Baseline treatment we expected minimal price volatility as the fixed expected marginal sanction would establish a price ceiling when abatement costs were at higher levels.

In the second and third treatments, we attempted to motivate full compliance by tying elements of the enforcement strategy to average permit prices in each round. The Audit treatment had a fixed per-unit penalty, but the monitoring probability varied directly with the average trading price. We parameterized this treatment so that, in theory, the resulting expected marginal penalty in every round would be roughly $10 \%$ higher than the average trading price, with the expectation that this would be sufficient to motivate full compliance by all subjects. With perfect compliance, there would be no emissions volatility - all the volatility in abatement costs would be transmitted to the permit price and monitoring effort.

To eliminate the transmission of risk to monitoring effort, the Fine treatment had a fixed 0.5 monitoring probability, but the per-unit penalty was tied directly to the average trading price in a period. We parameterized the Fine treatment so that the resulting expected marginal penalty would again be roughly $10 \%$ higher than the average trading price. Again, we expected full compliance, zero emissions volatility, and significant price volatility. Confirmation of these hypotheses would suggest that policymakers can maintain compliance in an emissions market and prevent the transmission of abatement cost volatility to enforcement costs by tying the marginal sanction directly to observed permit prices. In addition, tying sanctions to prices is likely to be easier to implement than tying monitoring to prices, which we discuss later in the paper. Thus, tying sanctions to prices has both theoretical and practical advantages over tying monitoring to prices.

Our results support some of our hypotheses, but not all. As expected, there were significant violations and emissions volatility under the Baseline treatment. However, violations 
and emissions volatility were not as high as predicted. Consequently, permit prices were higher and significantly more volatile than expected. That the subjects did not violate their permits as often as predicted is consistent with other work in this area (e.g., Stranlund et al. 2013, Raymond and Cason 2011).

Tying monitoring to average permit prices effectively eliminated noncompliance as the model predicts. In addition, emissions volatility was not significantly different than zero, but permit price volatility was significantly higher. This result confirms our hypothesis that making enforcement responsive to price volatility by tying monitoring to permit prices can eliminate noncompliance, but allows the transmission of abatement cost risk to monitoring effort.

Tying the sanction to average permit prices in the Fine treatment was less successful in inducing compliance. As hypothesized, violations and emissions volatility were significantly lower than under the Baseline; however, in theory both should be eliminated. Instead, we observed significant violations and emissions volatility in this treatment.

After analyzing the results from our original experiments, we conducted another treatment (High Fine) that involved tying the sanction to permit prices, but increased the markup so that the expected marginal penalty was $50 \%$ higher than the average price in a period. Our intention was to examine whether a substantial increase in the permit violation sanctions could eliminate noncompliance. Although violations were reduced in the High Fine treatment, they still were not eliminated. It is clear that subjects reacted differently to price-induced changes in monitoring than sanctions, although it is not clear why. Despite the theoretical and practical advantages of tying sanctions to permit prices, this strategy resulted in significant noncompliance whereas tying monitoring to prices did not.

In addition, we observed a small but non-trivial number of subjects who sold permits at very low prices, presumably attempting to reduce the expected marginal penalty. We demonstrate that this behavior could not have been individually rational, and hence may be unlikely in naturally-occurring emissions markets. However, it does raise the concern that tying permit violation penalties to going permit prices may motivate attempts to manipulate pricing. It may be possible to address these concerns with simple policy fixes. For example, imposing a minimum penalty or basing the penalty on the median trading price instead of the average price should eliminate the influence of permit sales at low prices on the resulting enforcement strategy. 
The rest of the paper proceeds as follows. In the next section we present the design of our experiments, including a sketch of the theory that motivates our work. In the third section we present our results. In the fourth section we conclude with a discussion of the relevance of our results for designing emissions markets.

\section{Experimental Design}

\subsection{Theory}

Our experimental design is based on a static model of emissions trading under regulatory uncertainty about firms' abatement costs with the possibility of noncompliance. An industry is composed of $n$ heterogeneous risk-neutral firms that emit a uniformly mixed pollutant. Firm $i$ emits $q^{i}$ units of the pollutant. Its abatement cost function is $c^{i}\left(q^{i}, u\right)$, which is strictly convex in $q^{i}$ and reaches a minimum at $\bar{q}^{i}$. Emissions control will limit each firm's emissions below this level so that the firm's marginal abatement cost function, $-c_{q}^{i}\left(q^{i}, u\right)$, is strictly decreasing for $q^{i}<\bar{q}^{i}$. The variable $u$ captures random shocks that affect the abatement costs of all firms. It has zero expectation and support $[\underline{u}, \bar{u}]$. Each firm's marginal abatement cost function is strictly increasing in $u$.

Under a simple emissions trading scheme, $L<\sum_{i=1}^{n} \bar{q}^{i}$ permits are distributed to the firms (free-of-charge), with $l_{0}^{i}$ permits going to firm $i$. Permit trades take place at price $p$ and firm $i$ holds $l^{i}$ permits when trading is complete. A firm is compliant if it holds enough permits to cover its emissions, that is, $q^{i} \leq l^{i}$, and it is noncompliant if $q^{i}>l^{i}$. To check for compliance, firms are monitored randomly with probability $m$. If a violation is discovered a penalty of $s$ per unit of violation $v^{i}=q^{i}-l^{i}$ is imposed on the firm. An equilibrium in the permit market requires that the price of permits not exceed the expected marginal penalty (i.e., $p \leq m s$ ), otherwise firms would sell all of their permits and be fully noncompliant.

Assuming that each firm chooses positive emissions and holds a positive number of permits, firm $i$ 's objective is to choose its emissions and permit holdings to minimize $c^{i}\left(q^{i}, u\right)+$ $p\left(l^{i}-l_{0}^{i}\right)+m s\left(q^{i}-l^{i}\right)$, subject to $v^{i}=q^{i}-l^{i} \geq 0$. Imposing the non-negativity constraint on a firm's violation follows from the fact that it will never have an incentive to be over-compliant in this model. If we let $\mathcal{L}^{i}$ denote the Lagrange equation for the firm's problem and we let $\lambda^{i}$ 
denote the multiplier attached to the constraint $q^{i}-l^{i} \geq 0$, the first-order conditions for a solution to the firm's problem are:

$$
\begin{aligned}
& \mathcal{L}_{q}^{i}=c_{q}^{i}\left(q^{i}, u\right)+m s-\lambda^{i}=0 \\
& \mathcal{L}_{i}^{i}=p-m s+\lambda^{i}=0 \\
& \mathcal{L}_{\lambda}^{i}=q^{i}-l^{i} \geq 0, \lambda^{i} \geq 0, \lambda^{i}\left(q^{i}-l^{i}\right)=0 .
\end{aligned}
$$

Because the constraint $v^{i}=q^{i}-l^{i} \geq 0$ is linear and the firm's objective is strictly convex, these conditions are necessary and sufficient to identify a firm's optimal choices of emissions, permit demand, and violation level.

Malik (1990) was the first to demonstrate that under random monitoring, a competitive permit market will distribute individual abatement choices so that aggregate abatement costs are minimized, given the level of aggregate abatement actually achieved. ${ }^{3}$ To see this, combine (1) and (2) to obtain $p=-c_{q}^{i}\left(q^{i}, u\right)$, which is the familiar rule that competitive firms will choose their emissions to equate the permit price to their marginal abatement costs. In turn, marginal abatement costs will be equal across firms, which form the necessary conditions for minimizing industry aggregate abatement costs, given the level of emissions that results in equilibrium. Moreover, the equilibrium permit price is equal to the minimum aggregate abatement cost function. That is, letting $Q$ denote aggregate emissions, in a permit market equilibrium we have

$$
p=-C_{Q}(Q, u)
$$

where

$$
C(Q, u)=\min _{\left\{q^{i}\right\}_{i=1}^{n}} \sum_{i=1}^{n} c^{i}\left(q^{i}, u\right), \text { subject to } \sum_{i=1}^{n} q^{i}=Q
$$

Note that (4) implicitly defines aggregate emissions in terms of permit prices as $Q=Q(p, u)$. Since firms' marginal abatement costs are increasing in the random variable $u$, the industry's aggregate marginal abatement cost function and aggregate emissions are increasing in $u$.

\footnotetext{
${ }^{3}$ With laboratory emissions markets, Stranlund et al. (2013) found that permit markets allocated individual emissions control cost-effectively, despite imperfect enforcement and significant violations.
} 
From (2) and (3) it is straightforward to see that a firm is compliant if $p<m s$. Note that this condition applies for every firm. It also applies regardless of the risk preferences of firm managers (Malik 1990, Stranlund 2008). Therefore, using (4), if $p=-C_{Q}(L, u)<m s$, then all firms are compliant and aggregate emissions equal the permit supply. However, assuming the $m s$ is fixed for the time being, define a level of the random variable $u$, call it $u^{c}$, such that $-C_{Q}\left(L, u^{c}\right)=m s$. For realizations of $u \leq u^{c}$, firms are fully compliant so that $Q(p, u)=L$ and the permit price is determined by $p=-C_{Q}(L, u)$. However, for $u>u^{c},-C_{Q}(L, u)>m s$. Since the permit price cannot rise above $m s$, aggregate emissions must increase beyond the permit supply to satisfy $p=-C_{Q}(Q, u)=m s$. Aggregate violations in these cases are $V=Q(m s, u)-$ L. Notice how abatement cost risk is transmitted to violations, which, in turn, transmits the risk to sanctioning effort and its costs. ${ }^{4}$

Violations are eliminated for all realizations of $u$ if the expected marginal penalty satisfies $-C_{Q}(L, \bar{u}) \leq m s$, because then the permit price would always be less than the expected marginal penalty. However, if $-C_{Q}(L, \bar{u})>m s$, then equilibrium permit prices, aggregate emissions and violations are as follows:

$$
\begin{gathered}
p= \begin{cases}m s, & \text { for } u \geq u^{c} \\
-C_{Q}(L, u), & \text { for } u \leq u^{c} ;\end{cases} \\
Q= \begin{cases}Q(m s, u), & \text { for } u \geq u^{c} \\
L, & \text { for } u \leq u^{c} ;\end{cases} \\
V= \begin{cases}Q(m s, u)-L, \text { for } u \geq u^{c} \\
0, & \text { for } u \leq u^{c} .\end{cases}
\end{gathered}
$$

It is clear that abatement cost risk can be transmitted to compliance outcomes (and aggregate emissions) in a permit market with a fixed enforcement strategy that is not sufficient to induce full compliance under all potential realizations of abatement costs. This transmission of risk is costly for several reasons. First, expected aggregate emissions will exceed the permitted

\footnotetext{
${ }^{4}$ Although we can specify aggregate violations uniquely when $-C_{Q}(L, u)>m s$, we are not able to specify unique individual violations because $m s$ is a constant.
} 
cap, leading to higher expected pollution damage. Second, the potential volatility in aggregate emissions is costly if the damage from pollution is strictly convex. Third, abatement cost risk increases the expected costs of sanctioning noncompliant firms. However, recall that Montero (2002) showed how constructing a policy that resulted in permit violations if abatement costs turned out to be high could be efficient because it also reduces expected abatement costs.

In this paper we do not specify the optimal use of imperfect enforcement under uncertainty about abatement costs. See Montero (2002) and Stranlund and Moffitt (2014) for this calculation. Instead, we are interested in how abatement cost risk is transmitted to compliance choices and how it might be controlled. We can stop the transmission of abatement cost risk to compliance in a variety of ways. One possibility that we already mentioned is to set the expected marginal penalty high enough so that $-C_{Q}(L, \bar{u}) \leq m s$. With this strategy the expected marginal penalty would exceed the permit price under all circumstances. However, the market is overmonitored, because $p<m s$ for realizations of $u$ below $\bar{u}$, which implies that monitoring (and monitoring costs) could be reduced so that $p=m s$ without affecting the firms' compliance choices or the market equilibrium.

A strategy that can stop the transmission of abatement cost risk to compliance choices and would limit the over-monitoring problem is to tie the monitoring probability directly to within-period variation in the permit price. That is, the monitoring probability could be set so that $m(p)=\alpha p$, where $\alpha \geq 1 / s$. This guarantees full compliance, because the expected marginal penalty never falls below the going permit price (i.e., $m(p) s=\alpha p s \geq p$ ). In turn, full compliance implies that the market equilibrium, given the realization of $u$, is described by $p=$ $-C_{Q}(L, u)$. Moreover, choosing $\alpha$ to be slightly above $1 / s$ eliminates the over-monitoring problem associated with fixed monitoring. Although this strategy prevents the transmission of abatement cost risk to compliance choices with minimal monitoring, it clearly transmits the risk to monitoring effort and its costs.

A final possibility is to tie the sanction $s$ to the permit price. Choosing $s=\beta p$ so that $\beta \geq 1 / m$ guarantees full compliance with constant monitoring, because $m s=m \beta p \geq p$. In the market equilibrium, $p=-C_{Q}(L, u)$ so that abatement cost risk is transmitted to the permit price, but then is absorbed by the penalty. Thus, like tying monitoring to prices, tying the sanction to prices can eliminate the transmission of abatement cost risk to compliance choices and the costs of sanctioning noncompliant firms. However, tying the sanction to prices has the additional 
advantage of eliminating the transmission of abatement cost risk to monitoring effort and its costs.

In addition, tying the sanction to prices may be much easier to implement in practice than tying monitoring to prices. While it seems straightforward to communicate to firms how an administrative penalty for permit violations will change with permit prices, the difficulty of specifying monitoring probabilities will make it difficult to convey how this probability is to change with permit prices. In practice, it is unlikely that regulators would attempt to determine and communicate a menu of monitoring probabilities. Instead, regulators could specify different monitoring strategies that depend on permit prices, with strategies that produce closer observation of firms' emissions implemented with higher permit prices. However, changing monitoring strategies as prices change may also be much more difficult than changing penalties. Monitoring strategies may be relatively inflexible because of inflexible agency budget allocations. Moreover, monitoring strategies may be inflexible because they are tied to specific monitoring technologies, for example, automated emissions reporting and verification procedures linked to continuous emissions monitoring systems that are used in some emissions markets. In contrast, changing sanctions as prices change can be done automatically. ${ }^{5}$

A potential problem with either tying monitoring or the penalty to permit trading prices to achieve full compliance is that there is a zero-price equilibrium with maximal violations in addition to the positive-price, full-compliance equilibria described above. With either monitoring or the penalty tied directly to the permit price as described above, at a zero price the expected marginal penalty is $m s=0$. Moreover, since there is a zero price for emissions and essentially no enforcement, each firm chooses emissions $\bar{q}^{i}$ that it would choose in a completely unregulated setting. We can show that this outcome is an equilibrium by showing that no firm is willing to buy a permit at a positive price. Suppose that a potential permit buyer has a violation $v^{i} \geq 0$. If $v^{i}=0$, then purchasing an additional permit at a positive price would mean that the

\footnotetext{
${ }^{5}$ Another implementation issue is which price should be used to determine enforcement. In our experiments we use the average trading price in a period, but we discuss how the median price may be a better choice because it is not sensitive to outlier trades. Choosing a price from a different part of the price distribution may also have merit. As noted earlier, in the proposed but not enacted U.S. Clear Skies Initiative (2003) and the American Clean Energy and Security Act (2009), sanctions were to be tied to the clearing price of a recent permit auction. Most of the pilot programs of China's $\mathrm{CO}_{2}$ emissions market set penalties at three to five times the average market price of $\mathrm{CO}_{2}$ allowances.
} 
firm is spending money to become over-compliant, which it has no incentive to do. If $v^{i}>0$, then purchasing an additional permit reduces the firm's violation by one unit. However, purchasing an additional permit at a positive price increases the expected penalty of any remaining violation, which were costless before the purchase. Therefore, no permit trades at a positive price will take place from a zero-price outcome, implying that this outcome is a potential equilibrium.

While possible, achieving a zero-price equilibrium appears to require a significant degree of coordination because it requires all sellers to offer permits at a zero price. Nevertheless, that there is such an equilibrium, however remote the likelihood that it will be reached, might suggest that some permit sellers will offer permits at very low prices to reduce the expected marginal penalty.

We designed our experiments to examine the transmission of abatement cost risk under three enforcement strategies. The first involves a fixed enforcement strategy that is not sufficient to induce full compliance to investigate the transmission of abatement cost risk to compliance choices. The second enforcement strategy ties the monitoring strategy to observed permit prices to examine the possibility of stopping the transmission of abatement cost risk to compliance choices while absorbing the risk with monitoring effort. Our third strategy ties the sanction to observed prices in an attempt to prevent the transmission of abatement cost risk to both compliance choices and enforcement costs.

\subsection{Experiment Procedures}

Instead of framing our experiments as decisions to emit a pollutant we chose a more neutral frame in which subjects made a decision to produce a fictitious commodity. During each threeminute period, subjects simultaneously produced units of the good and traded in a discriminative price double auction for permits that conveyed the right to produce. Each group contained eight individuals evenly divided among four types that differed in terms of their production earnings, initial permit allocations and initial cash. Individual initial permit allocations and initial cash did not vary across time periods. ${ }^{6}$ The environment was static; each period was independent and there was no banking or borrowing of permits.

\footnotetext{
${ }^{6}$ The instructions are available in section 1 of the online Appendix.
} 
In line with our theoretical model, we introduced regulatory uncertainty by varying the subjects' marginal production earnings each period. There were three possible marginal earnings schedules for each subject type, Low (L), Medium (M) and High (H), shown in Table 1. These earnings schedules are individual permit demand schedules, and are equivalent to the individual marginal abatement costs we used to develop the theory in the previous section. We will refer to these schedules as permit demand schedules from here on. For each subject type, the different demand schedules are parallel — the High demand schedule is E\$69 above the Medium demand schedule for each unit of production and the Low demand schedule is E\$69 below the Medium demand schedule. A total of 24 permits were supplied to the market in each period. Player types A and B started each round with zero permits, and player types $\mathrm{C}$ and D started each round with six permits each. Subjects also differed in their initial cash endowments. Player types A and B received $\mathrm{E} \$ 2000$ at the start of each period, while types $C$ and $D$ received $E \$ 500$ at the start of each period.

Table 1. Marginal Production Earnings/Individual Permit Demand Schedules

\begin{tabular}{|c|c|c|c|c|c|c|c|c|c|c|c|c|}
\hline \multirow{3}{*}{$\begin{array}{c}\text { Units } \\
\text { Produced }\end{array}$} & \multicolumn{12}{|c|}{ Subject Type } \\
\hline & \multicolumn{3}{|c|}{$\mathbf{A}$} & \multicolumn{3}{|c|}{$\mathbf{B}$} & \multicolumn{3}{|c|}{$\mathbf{C}$} & \multicolumn{3}{|c|}{ D } \\
\hline & $\mathrm{L}$ & $\mathrm{M}$ & $\mathrm{H}$ & $\mathrm{L}$ & $\mathrm{M}$ & $\mathrm{H}$ & $\mathrm{L}$ & $\mathrm{M}$ & $\mathrm{H}$ & $\mathrm{L}$ & $\mathrm{M}$ & $\mathrm{H}$ \\
\hline 1 & 261 & 330 & 399 & 251 & 320 & 389 & 241 & 310 & 379 & 231 & 300 & 369 \\
\hline 2 & 241 & 310 & 379 & 231 & 300 & 369 & 221 & 290 & 359 & 211 & 280 & 349 \\
\hline 3 & 221 & 290 & 359 & 211 & 280 & 349 & 201 & 270 & 339 & 191 & 260 & 329 \\
\hline 4 & 201 & 270 & 339 & 191 & 260 & 329 & 181 & 250 & 319 & 171 & 240 & 309 \\
\hline 5 & 181 & 250 & 319 & 171 & 240 & 309 & 161 & 230 & 299 & 151 & 220 & 289 \\
\hline 6 & 161 & 230 & 299 & 151 & 220 & 289 & 141 & 210 & 279 & 131 & 200 & 269 \\
\hline 7 & 141 & 210 & 279 & 131 & 200 & 269 & 121 & 190 & 259 & 111 & 180 & 249 \\
\hline 8 & 121 & 190 & 259 & 111 & 180 & 249 & 101 & 170 & 239 & 91 & 160 & 229 \\
\hline 9 & 101 & 170 & 239 & 91 & 160 & 229 & 81 & 150 & 219 & 71 & 140 & 209 \\
\hline 10 & 81 & 150 & 219 & 71 & 140 & 209 & 61 & 130 & 199 & 51 & 120 & 189 \\
\hline
\end{tabular}

In each eight-person group, there were two subjects of each type. The sub-headings L, M, H refer to Low, Medium and High demand periods.

Each session lasted 15 periods, with marginal production earnings varying in each period. Subjects were told at the start of each session that production earnings would vary such that they would face High, Medium and Low production earning in a random order in each set of three periods. The sequence of production earnings for each session is in Table 2. Despite being 
uncertain about the path of the shocks to their demand schedules, the subjects knew which demand schedule they faced at the start of each period.

\section{Table 2. Sequence of production earnings for each session}

\begin{tabular}{lccccccccccccccc}
\hline Period & 1 & 2 & 3 & 4 & 5 & 6 & 7 & 8 & 9 & 10 & 11 & 12 & 13 & 14 & 15 \\
Production Earnings & L & M & H & L & H & M & M & L & H & M & H & L & H & L & M \\
\hline
\end{tabular}

During a period, subjects produced each unit of the good by initiating a "production process" that took ten seconds to complete. Production took place over 2.5 minutes, which was more than sufficient for each subject to produce up to their capacity constraint of 10 units. The market for permits was open for that entire time plus an additional 30 seconds to allow the subjects to adjust their permit holdings after production was completed. This sort of reconciliation period is common in real emissions markets.

At the end of a period, subjects' production choices were audited with a probability that, depending upon treatment, was either known at the beginning of the session and was the same in every period, or it varied according to the average price of permits traded in the period, as explained in the next subsection. If a subject's permit violation was discovered, the subject received a fixed sanction for each unit of production in excess of his or her permit holdings. This per-unit sanction was either a known constant throughout a session, or varied with the average trading price in a period.

A total of 128 subjects (four groups of eight subjects in each of four treatments) were recruited from the general student population at the University of Massachusetts Amherst. Subjects participated in a training session on one evening and a data session on a subsequent day. For each group, the treatment used in the training session was identical to the data session, but with different parameters. At the start of the training session, subjects were paid $\$ 5$ for agreeing to participate and showing up on time. They were given an additional \$20 if they returned for the data session, in which they could earn additional money. Subjects earned experimental dollars (E\$) that were converted to US dollars at a pre-announced exchange rate $(\mathrm{E} \$ 1600=\mathrm{US} \$ 1)$. Average earnings in the data sessions ranged between $\$ 15.94$ and $\$ 33.94$, with a mean of \$25.99 $(\sigma=4.21)$. Data sessions lasted approximately one hour. 


\subsection{Treatments and Hypotheses}

It is helpful to use Figure 1 to explain the treatments in our experiments. The lines labeled High Demand, Medium Demand, and Low Demand in the figure are aggregate permit demand schedules derived from the individual permit demand schedules in Table 1. In each treatment, 24 permits were supplied to the market. Consequently, assuming perfect competition and full compliance under each demand state, the equilibrium prices would be $\mathrm{E} \$ 201, \mathrm{E} \$ 270$ and $\mathrm{E} \$ 339$ under Low Demand, Medium Demand and High Demand states, respectively. However, in our Baseline treatment, we chose a fixed monitoring probability of 0.5 and a sanction of $E \$ 440$, so the expected marginal sanction was $\mathrm{E} \$ 220$. While this enforcement strategy is high enough to induce full compliance under the Low Demand state, under the Medium and High Demand states the equilibrium permit price rises to the expected marginal penalty of $\mathrm{E} \$ 220$, and aggregate emissions increase to 41-44 units and 68 units, respectively (which corresponds to aggregate violations of 17-20 and 44 units). Notice how abatement cost uncertainty is transmitted primarily to compliance choices in the Baseline treatment through changes in emissions and violations.

Figure 1. Aggregate permit demand shocks and equilibrium prices.

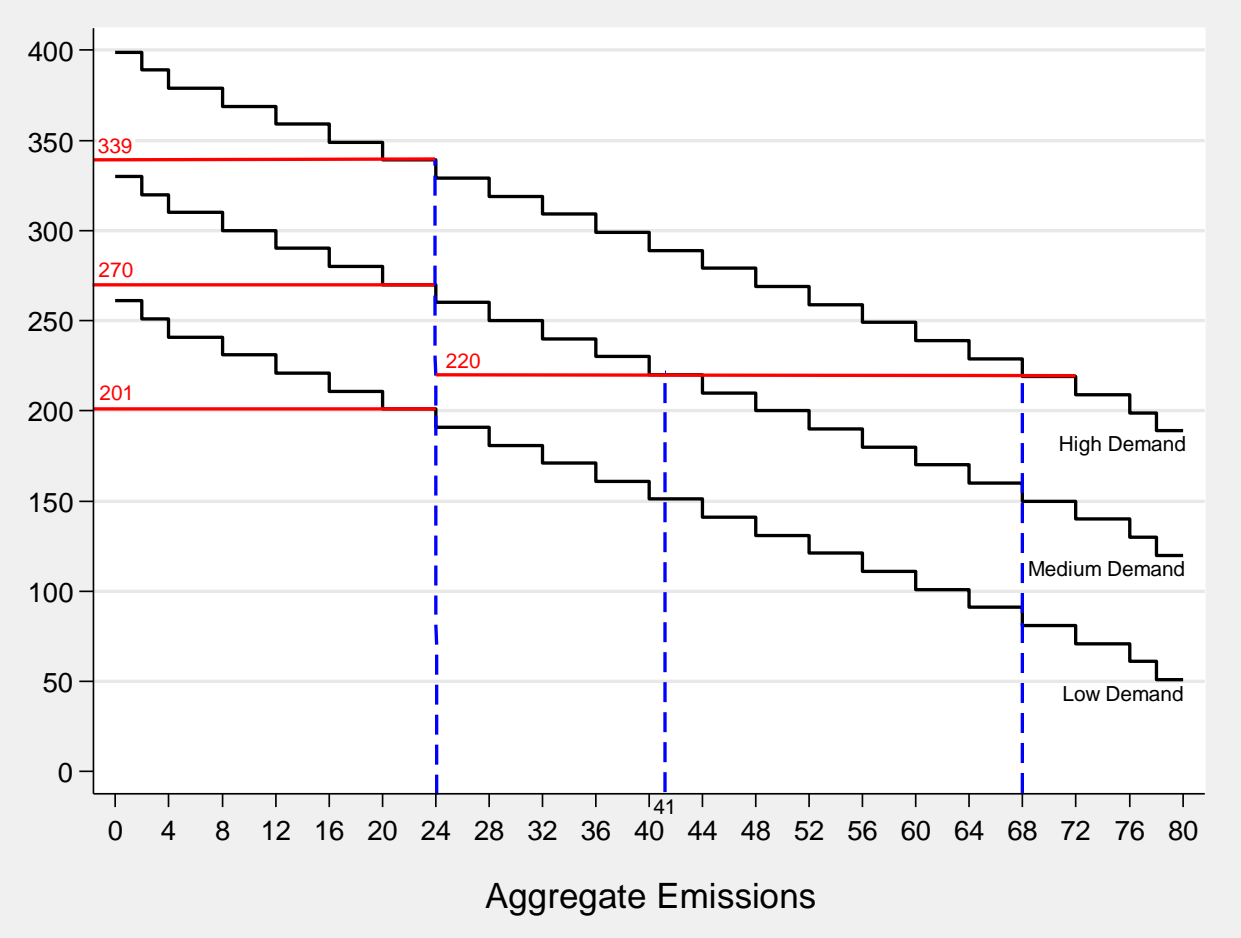


We conducted three additional treatments with alternative enforcement strategies that were designed to shift abatement cost risk from the subjects' compliance choices to the permit price and either the monitoring probability or the violation penalty. In our Audit treatment we tied the monitoring probability directly to permit trading prices in an attempt to induce full compliance as described in section 2.1. Specifically, we fixed the sanction at $\mathrm{E} \$ 440$ per unit, but we allowed the monitoring probability to vary according to $m=(0.0025) p^{a}$, where $p^{a}$ is the average of all the trading prices in a period. ${ }^{7}$ Under the assumption that the average trading price in a period is equal to the predicted equilibrium permit price, this enforcement strategy motivates full compliance under all demand states. In Table 3, we provide the equilibrium price and the monitoring probability $(m)$, sanctions $(s)$ and expected marginal sanction $(m s)$ under each demand state for each treatment. Note how the monitoring probability in the Audit treatment increases with the demand level and, given the constant E\$440 unit sanction, how the expected marginal sanction exceeds the equilibrium permit price by about $10 \%$ in each demand state.

In our Fine treatment, we tied the sanction to average permit prices. The monitoring probability was set at a constant 0.50 , but the unit sanction varied according to $s=(2.24) p^{a}$. Again, assuming that the average price in a period is equal to the predicted equilibrium price, Table 3 shows the predicted marginal sanction and expected marginal sanction under each of the demand states in the Fine treatment. The predicted expected marginal sanction exceeds the equilibrium permit price by $12 \%$ for every demand state, so subjects should be fully compliant despite the volatility in demand. The sanction absorbs the demand volatility so that monitoring and compliance choices remain constant. ${ }^{8}$

\footnotetext{
${ }^{7}$ In the Audit treatment, at the start of each period, the monitoring probability was set initially at 0.25 , but was updated and displayed in real time for the subjects as trades occurred.

${ }^{8}$ We originally intended our design to produce the same predicted expected marginal penalties in the Audit and Fine treatments, but a software coding error made the expected marginal penalty about $2 \%$ higher in the Fine treatments. This small difference does not change any of the price and quantity predictions in these treatments.
} 
Table 3. Enforcement strategies by treatment and demand state

\begin{tabular}{|c|c|c|c|c|c|c|c|c|c|c|}
\hline \multirow[b]{2}{*}{ Demand } & \multirow[b]{2}{*}{ Price } & \multicolumn{3}{|c|}{ Audit } & \multicolumn{3}{|c|}{ Fine } & \multicolumn{3}{|c|}{ High Fine } \\
\hline & & $m^{\text {a }}$ & $S$ & $m s$ & $m$ & $S$ & $m s$ & $m$ & $s$ & $m s$ \\
\hline Low & 201 & 0.50 & 440 & 220.0 & 0.50 & 450 & 225.0 & 0.50 & 603 & 301.5 \\
\hline Medium & 270 & 0.68 & 440 & 299.2 & 0.50 & 605 & 302.5 & 0.50 & 810 & 405.0 \\
\hline High & 339 & 0.85 & 440 & 374.0 & 0.50 & 759 & 379.7 & 0.50 & 1017 & 508.5 \\
\hline
\end{tabular}

${ }^{\mathrm{a}}$ In the experiments $m$ was displayed to two decimal places while $s$ was displayed as an integer.

After reviewing the results from the first three treatments, we decided to add one last treatment, labeled High Fine, which was identical to the Fine treatment except that the markup for the sanction was higher. The sanction was set at three times the average permit price in a period; that is, $s=(3.0) p^{a}$. With this strategy, the predicted expected marginal penalty exceeds the equilibrium permit price by $50 \%$ in every demand state. ${ }^{9}$

Our experimental design allows us to test the main implications of the model in the previous section. In particular, we test whether an enforcement strategy that ties either monitoring or sanctions to prevailing permit prices will induce full compliance, and in doing so, whether it will transmit uncertainty about abatement costs from emissions and compliance choices to permit prices and enforcement. More specifically, we should observe significant violations in the Baseline treatment in the Medium and High demand states, resulting in significant volatility in aggregate emissions over the 15 periods of these experiments, but little volatility in permit prices. In contrast, under the Audit, Fine and High Fine treatments, we should observe full compliance, insignificant volatility in aggregate emissions, but significant volatility in permit prices. Of particular interest is whether subjects will be fully compliant under the Fine and High Fine treatments, as this would support the theoretical argument that tying sanctions to permit prices can completely stop the transmission of abatement cost risk to enforcement costs.

\section{Results}

The results of our experiments concern the effects of the different enforcement strategies on the levels and between-period volatility of group emissions, group violations, and permit prices. The

\footnotetext{
${ }^{9}$ In both the Fine and High Fine treatments, the unit sanction was set initially at E $\$ 220$, but was updated in real time as trades occurred.
} 
focus on group outcomes is consistent with our theory and hypotheses, which concern the effects of alternative enforcement strategies on aggregate market outcomes.

\section{Emissions, violations, and emissions volatility}

In the top part of Table 4, we present the results of tests of the null hypotheses that estimated group emissions, group violations, and emissions volatility are equal to their predicted values. To conduct these tests we used linear random effects models of the form $y_{g t}=\alpha_{0}+x_{g t} \beta+v_{g}+$ $\varepsilon_{g t}$, with aggregate emissions, violations, and emissions volatility as the dependent variables. Random effects for each group are $v_{g} \sim N\left(0, \sigma_{v}^{2}\right), \varepsilon_{g t} \sim N\left(0, \sigma_{\varepsilon}^{2}\right)$ is the idiosyncratic error term, and $x_{g t}$ is a vector of independent variables for group $g$ in period $t$. Emissions volatility for group $g$ is the mean absolute difference in the group's aggregate emissions between periods $t$

and $t-1$; that is, $\sum_{t=2}^{T=15}\left|Q_{g t}-Q_{g, t-1}\right| /(T-1)$. The independent variables include dummy variables for treatment, demand state, and their interactions. All significance tests are chi-square tests using these models. We do not present the regressions in this paper, but they are available in the online Appendix. Because we had some instances of over-compliance (i.e., subjects held excess permits), estimated group emissions volatility is not the same as estimated group violation volatility. However, they are close enough so we only report estimated group emissions volatility.

The lower part of the table presents the results of the tests of the null hypotheses that estimated group emissions and emissions volatility are equal between treatments. Treatment differences in group violations were nearly identical to emissions treatment differences, so we only report the emissions results.

Consistent with the model presented in the previous section, there were no significant violations in the Baseline treatment when demand was Low. As predicted, in both the Medium and High demand states violations were positive, however both violations and emissions were significantly lower than predicted. Consequently, emissions volatility was significantly lower as well. Estimated emissions under the Audit treatment were consistent with predictions. Hence, our predictions that there would be zero violations and zero emissions volatility in the Audit treatment are supported statistically. Of course, we made the same predictions for the Fine treatment, but the results in Table 4 reveal that emissions under this treatment were significantly 
higher than predicted in the Medium and High demand states. Hence, although the Fine treatment was designed to eliminate violations and emissions volatility, this did not occur.

Recall that we added the High Fine treatment to examine whether a higher markup for the permit violation penalty would eliminate the significant violations we observed in the Fine treatment. The results in Table 4 show that estimated violations under the High Fine treatment were lower than under the Fine treatment, although not significantly so. More to the point, violations under the High Fine treatment were still positive and significant in the Medium and High demand states.

Table 4. Aggregate emissions, violations, and emissions volatility ${ }^{\text {a }}$

\begin{tabular}{|c|c|c|c|c|}
\hline \multirow[b]{2}{*}{ Treatment } & \multicolumn{3}{|c|}{ Demand States } & \multirow[b]{2}{*}{ Volatility } \\
\hline & 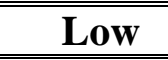 & Medium & High & \\
\hline \multicolumn{5}{|l|}{ Baseline } \\
\hline Estimated Emissions & $25.45^{b}$ & $32.85 * * *$ & $45.00 * * *$ & $13.38 * * *$ \\
\hline Predicted Emissions & 24.00 & $41.00-44.00^{\mathrm{c}}$ & 68.00 & 30.21 \\
\hline Estimated Violations & 2.50 & $9.15 * * *$ & $21.05^{* * *}$ & \\
\hline Predicted Violations & 0.00 & 17.00 & 44.00 & \\
\hline \multicolumn{5}{|l|}{ Audit } \\
\hline Estimated Emissions & 26.40 & 25.20 & 25.10 & 2.02 \\
\hline Predicted Emissions & 24.00 & 24.00 & 24.00 & 0.00 \\
\hline Estimated Violations & 2.80 & 1.50 & 1.30 & \\
\hline Predicted Violations & 0.00 & 0.00 & 0.00 & \\
\hline \multicolumn{5}{|l|}{ Fine } \\
\hline Estimated Emissions & 26.95 & $29.10 * *$ & $31.65^{* * *}$ & $4.69 * * *$ \\
\hline Predicted Emissions & 24.00 & 24.00 & 24.00 & 0.00 \\
\hline Estimated Violations & $4.35 * *$ & $5.90 * * *$ & $8.10 * * *$ & \\
\hline Predicted Violations & 0.00 & 0.00 & 0.00 & \\
\hline \multicolumn{5}{|l|}{ High Fine } \\
\hline Estimated Emissions & 26.10 & $27.90 *$ & $28.65 * *$ & 3.39 \\
\hline Predicted Emissions & 24.00 & 24.00 & 24.00 & 0.00 \\
\hline Estimated Violations & 2.80 & $4.20 * *$ & $4.85^{* *}$ & \\
\hline Predicted Violations & 0.00 & 0.00 & 0.00 & \\
\hline \multicolumn{5}{|c|}{ Emissions Treatment Differences } \\
\hline Baseline-Audit & -0.95 & $7.65 * * *$ & $19.9 * * *$ & $11.36 * * *$ \\
\hline Baseline-Fine & -1.50 & 3.75 & $13.35 * * *$ & $8.68 * * *$ \\
\hline Baseline-High Fine & -0.65 & $4.95 *$ & $16.35^{* * *}$ & $9.98 * * *$ \\
\hline Audit-Fine & -0.55 & -3.90 & $-6.55 * *$ & -2.67 \\
\hline Audit-High Fine & 0.30 & -2.70 & -3.55 & -1.37 \\
\hline Fine-High Fine & 0.85 & 1.20 & 3.00 & 1.30 \\
\hline
\end{tabular}

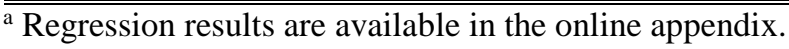


${ }^{\mathrm{b}}$ Estimated emissions minus the supply of permits (24) does not generally equal estimated violations because there were some instances of overcompliance by small amounts.

${ }^{c}$ Predicted emissions in the Medium demand state under the Baseline treatment is the range 41-44. Predicted and estimated violations, as well as the tests to compare estimated emissions and violations to their predicted values are based on the lower bound of this range (41).

$* * * p<0.01$, ** $p<0.05, * p<0.1$.

The differential compliance responses in the Audit treatment compared to the Fine and High Fine treatments are puzzling. One might suspect that non-neutral attitudes toward risk could explain the different responses to changes in monitoring frequency and to changes in the sanctions. However, Stranlund (2008) showed that while differential risk preferences can drive differences in the violation choices of noncompliant firms, differential risk preferences do not affect the decision of whether to comply. Firms would be compliant if the expected marginal penalty is above the going permit price regardless of whether their managers are risk averse, risk neutral or risk seeking. Since our Audit, Fine and High Fine treatments were designed to induce full compliance by making the expected marginal penalty higher than average permit prices, it is unlikely that the differential compliance responses in these treatments can be explained by differences in risk preferences.

Noncompliance in the Fine and High Fine treatments reduces expected payoffs, so one may wonder whether this behavior would persist with more experience. We do not observe a decline in noncompliance over time in our data. It is possible, however, that modified Fine and High Fine treatments with more rounds and detailed feedback to make the losses more transparent could mitigate noncompliance as subjects gain a better understanding of the consequences of permit violations.

Although violations were not eliminated in the Fine and High Fine treatments, they tended to be significantly lower than in the Baseline treatment in the Medium and High demand states. (The violation comparisons are not shown in Table 4, because they were close to the emissions treatment differences). The impacts of the Fine and High Fine treatments on reducing violations were not as great as predicted because violations were lower than predicted in the Baseline treatment, and higher than predicted in the Low and High Fine treatments. Finally, note that emissions volatility was higher in the Baseline treatment than in the other three treatments. This is what the model predicts. Thus, compared to a fixed enforcement strategy with the potential for significant noncompliance, tying enforcement strategies to permit prices can reduce 
emissions volatility. We will see shortly that the reductions is emissions volatility is associated with an increase in price volatility ${ }^{10}$.

\section{Permit prices}

Similar to the structure of Table 4, in the top part of Table 5 we present estimated and predicted prices by treatment and demand state, as well as estimated price volatility by treatment. Average within-period prices, $p_{g t}^{a}=(1 / I) \sum_{i=1}^{I} p_{i g t}$, are calculated as the mean of all transactions ( $I$ total transactions indexed by $i$ ) for a given group $g$ in period $t$. Price volatility, $\sum_{t=2}^{T} \mid p_{g t}^{a}-$ $p_{g, t-1}^{a} \mid /(T-1)$, is the mean of the absolute differences in the average price for group $g$ between periods $t$ and $t-1$. In the lower part of Table 5, we present differences in estimated prices between treatments for each demand state, as well as differences in price volatility between treatments. In the top part of the Table 5 we indicate the results of tests of the null hypotheses that estimated prices and price volatility are equal to their predicted values, while the lower part of the table indicates results of the tests of the null hypotheses that estimated prices and price volatility are equal between treatments. To conduct hypothesis tests, we use linear random effects models with the same structure as the emissions and violation regressions except that the dependent variables are average prices and price volatility. ${ }^{11}$ These regressions are available in the online Appendix.

One important result in Table 5 is that estimated prices in the Baseline treatment were much higher than predicted values. This result is consistent with the lower-than-predicted violations in this treatment - subjects in this treatment did not violate their permits as much as predicted so demand for permits was greater, which led to higher permit prices. Note that the higher-than-predicted prices in this treatment are associated with significantly greater price volatility than predicted.

\footnotetext{
${ }^{10} \mathrm{We}$ also tested for time trends and learning across periods. See the online appendix. Overall, no statistically significant differences in emissions, violations were detected across periods in the various treatments.

${ }^{11}$ We also tested for potential differences in within-period permit price dispersion, calculated as the mean absolute difference between each trading price and that period's average price. We found no statistically significant treatment differences in price dispersion among the treatments.
} 
Table 5. Average within-period permit prices and between-period price volatility ${ }^{\text {a }}$

\begin{tabular}{|c|c|c|c|c|}
\hline \multirow[b]{2}{*}{ Treatment } & \multicolumn{3}{|c|}{ Demand States } & \multirow[b]{2}{*}{ Volatility } \\
\hline & Low & Medium & High & \\
\hline \multicolumn{5}{|l|}{ Baseline } \\
\hline Estimated Prices & $226.42 * * *$ & $279.24 * * *$ & $319.80 * * *$ & $66.94 * * *$ \\
\hline Predicted Prices & 201.00 & 220.00 & 220.00 & 12.21 \\
\hline \multicolumn{5}{|l|}{ Audit } \\
\hline Estimated Prices & 212.15 & $290.43 * *$ & 342.55 & 89.57 \\
\hline Predicted Prices & 201.00 & 270.00 & 339.00 & 93.64 \\
\hline \multicolumn{5}{|l|}{ Fine } \\
\hline Estimated Prices & 213.97 & $279.01 * *$ & 338.51 & 90.16 \\
\hline Predicted Prices & 201.00 & 270.00 & 339.00 & 93.64 \\
\hline \multicolumn{5}{|l|}{ High Fine } \\
\hline Estimated Prices & 196.87 & $251.84 * *$ & $306.10 * * *$ & $80.04 * *$ \\
\hline Predicted Prices & 201.00 & 270.00 & 339.00 & 93.64 \\
\hline \multicolumn{5}{|c|}{ Price Treatment Differences } \\
\hline Baseline-Audit & 14.27 & -11.19 & $-22.75^{*}$ & $-22.63^{* *}$ \\
\hline Baseline-Fine & 12.44 & 0.23 & -18.71 & $-23.22 * *$ \\
\hline Baseline-High Fine & $29.55 * *$ & $27.4 * *$ & 13.71 & -13.10 \\
\hline Audit-Fine & -1.83 & 11.42 & 4.04 & -0.59 \\
\hline Audit-High Fine & 15.28 & $38.59 * * *$ & $36.46 * * *$ & 10.12 \\
\hline Fine-High Fine & 17.11 & $27.18 * *$ & $32.42 * *$ & 9.53 \\
\hline
\end{tabular}

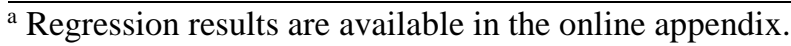

$* * * p<0.01, * * p<0.05, * p<0.1$.

Estimated prices and price volatility in the Audit and Fine treatments were typically close to predicted values (except under the Medium demand state). Consequently, as hypothesized, there were no significant differences in estimated prices and price volatility between the Audit and Fine treatments. Comparing the Baseline treatment to the Audit and Fine treatments, estimated prices were similar although we expected them to be far apart in the Medium and High demand states. This was due to higher than predicted prices under the Baseline treatment but prices that were similar to predictions in the Audit and Fine treatments.

Our model predicts that tying the enforcement strategies (monitoring or penalties) to average prices would increase permit price volatility, relative to the Baseline treatment, and this is exactly what we observe. However, this effect is not as strong as predicted because price 
volatility was significantly higher under the Baseline treatment than we expected. In the Audit, Fine and High Fine treatments, estimated price volatility was close to predicted values ${ }^{12}$.

Perhaps the most striking feature of the price results in Table 5 is that estimated prices under the Medium and High demand states in the High Fine treatment were significantly lower than predicted. These prices were also significantly lower than estimated prices in the Fine and Audit treatment, even though they were predicted to be the same. This phenomenon cannot be explained by differences in violation levels (i.e., permit demand) between predicted levels and between treatments. In fact, it suggests that there might have been attempts to push average prices down to reduce the noncompliance sanction.

In Table 6 we show the number of permit sales at very low prices - that is, less than or equal to $\mathrm{E} \$ 10$ - in each treatment. In addition, we show the number of subjects making these sales and their total violations. To examine the effects of low-price sales and violations on earnings, we compare the average earnings of subjects that sold permits under $\mathrm{E} \$ 10$ and violated versus everyone else. The last column of Table 6 shows the average earnings of subjects that never violated their permits. Given the small number of individuals who engaged in a low-price selling strategy and violated their permits, as well as the randomness of violation sanctions, the differences in earnings among the last three columns of Table 6 are not statistically significant. Nevertheless, the point estimates are intriguing. ${ }^{13}$

There was a small number of low-price sales by a single subject in each of the Baseline and Audit treatments. There were more permit sales at very low prices by several individuals in the Fine treatment, but a single individual made half of these trades. This subject had 18 violations (about $25 \%$ of the total) and made below average earnings for the treatment $(\mathrm{E} \$ 29,000)$. In the post-experiment survey, this individual clearly stated that he hoped to start a trend of low-price sales to reduce the expected penalty, but that this attempt failed because it relied on others following his lead, which did not happen. This is consistent with our theory,

\footnotetext{
${ }^{12}$ We also tested for the potential presence of time trends and learning across periods. See the online appendix. Overall, no statistically significant differences in prices or price volatility were detected across periods in the various treatments.

${ }^{13}$ We conducted robustness checks using different thresholds for how we define low-price permit sales, namely $\mathrm{E} \$ 20, \mathrm{E} \$ 30, \mathrm{E} \$ 50$ and E\$100. Qualitatively, our discussion here does not change with alternative low-price thresholds. We reproduce Table 6 in the online Appendix for the alternative price thresholds. In addition, we explored whether low price trades occurred in particular demand states or periods. The results (presented in the Appendix) suggest that neither demand state nor period explains low price trades..
} 
which admits a zero-price equilibrium with monitoring or sanctions tied to prices, but this outcome requires significant coordination among the traders.

Table 6: Sales below $\leq \mathbf{E} \$ 10$, violations and earnings

\begin{tabular}{lcccccc}
\hline \hline Treatment & $\begin{array}{c}\text { Number of } \\
\text { sales } \leq \mathbf{E \$ 1 0} \\
\text { (share of total } \\
\text { sales in } \\
\text { parenthesis) }\end{array}$ & $\begin{array}{c}\text { Number } \\
\text { of } \\
\text { subjects }\end{array}$ & $\begin{array}{c}\text { Total } \\
\text { violations }\end{array}$ & $\begin{array}{c}\text { Average total } \\
\text { earnings of } \\
\text { subjects with } \\
\text { sales } \mathbf{E} \mathbf{\text { \$10 }} \\
\text { that violated }\end{array}$ & $\begin{array}{c}\text { Average total } \\
\text { earnings of } \\
\text { all others }\end{array}$ & $\begin{array}{c}\text { Average total } \\
\text { earnings of } \\
\text { subjects that } \\
\text { never violated }\end{array}$ \\
\hline \hline Baseline & $2(0.23 \%)$ & 1 & $4(0.6 \%)$ & 34,518 & 33,511 & 29,886 \\
Audit & $5(0.59 \%)$ & 1 & $21(19 \%)$ & 24,493 & 31,492 & 32,407 \\
Fine & $10(1.16 \%)$ & 4 & $73(20 \%)$ & 33,361 & 30,661 & 31,685 \\
High Fine & $50(5.9 \%)$ & 9 & $129(54 \%)$ & 25,562 & 31,095 & 32,512 \\
\hline \hline
\end{tabular}

The number of low-price sales and subjects engaging in this behavior increased dramatically in the High Fine treatment, which contributed to the lower than predicted prices in this treatment. Notably, the nine individuals who made these low-price sales accounted for more than $50 \%$ of the violations in this treatment. On average, these individuals earned nearly E\$6000 (about US\$3.75) less than everyone else, and E\$7000 (US\$4.38) less than those subjects who never violated their permits. A deeper probe reveals that two subjects in two different groups were responsible for 41 of the 50 low-price sales $(82 \%)$ in the High Fine treatment. One of these subjects wrote in the post-experiment survey, "I saw that the penalty decreased when a sale that was lower than the average sale for permits was made... I was the one that kept selling my permits for $\$ 1$ to try to get people to catch what I was doing." ${ }^{14}$ Clearly, this subject was trying to lower the expected penalty by making $\mathrm{E} \$ 1$ sales hoping that others would follow suit. It did not pay off. This individual earned only E\$570 (about 36 cents) in the experiment. ${ }^{15}$

While some individuals attempted to reduce the expected marginal penalty by selling permits at very low prices, it is hard to imagine how they could have increased their expected payoffs with these attempts. A subject who sells a permit at a low price either increases the size

\footnotetext{
14 The other subject did not leave a comment in the post-experiment survey.

15 This subject made several poor decisions. He had 51 violations (about $40 \%$ of the total in the treatment) and was audited 13 times. Perhaps more damaging is that he was a type A subject, each of whom received no initial allocation of permits and were predicted to buy permits from other subjects. In fact, this individual bought permits in the $\mathrm{E} \$ 200-\mathrm{E} \$ 350$ range in early rounds only to sell some of them at $\mathrm{E} \$ 1$ in later rounds.
} 
of his or her violation, reduces his or her emissions, or reduces his or her excess permits (i.e., level of overcompliance). Selling permits at E\$10 or less to increase a violation could not increase expected earnings, because the expected marginal penalty never fell below E $\$ 200$ in the High Fine treatment and never below E\$150 in the Fine treatment. Selling permits at E\$10 or less while reducing emissions could not increase expected earnings, because the marginal emissions benefits for any player type or demand state was never below E\$50 (see Table 1). Finally, an individual who sold permits at E\$10 or less without changing his or her emissions or violations would be worse off, because trades were possible at substantially higher prices in all settings.

Still we should not simply ignore the temptation to sell permits at low prices when these prices determine sanctions. There may be simple fixes to the enforcement strategy to mitigate or eliminate this temptation. For example, consider imposing a minimum penalty, or penalty floor. This would eliminate the possibility of a zero-price equilibrium, and hence, may mitigate the temptation to make low-price sales to reduce the expected marginal penalty. Another possibility is to base the penalty on the median trading price instead of the average price. This does not eliminate the zero-price equilibrium, but it would eliminate the influence of outlier trades at very low prices. We think it is worthwhile to conduct modified Fine and High Fine experiments to test whether these simple adjustments can prevent attempts to reduce expected marginal penalties by selling permits at very low prices.

\section{Conclusions}

We have conducted experiments to investigate the transmission of abatement cost risk to compliance choices and enforcement strategies in emissions markets, and have found new results that have important implications for designing enforcement strategies for emissions markets.

- Random abatement cost shocks can result in substantial violations and emissions volatility under a fixed expected marginal penalty that is not sufficient to induce full compliance under all possible realizations of abatement costs. However, like other authors, we found that subjects did not violate their permits as much as the model predicted, and consequently, emissions volatility was significantly lower than hypothesized. 
- Tying monitoring to permit prices in the Audit treatment so that the expected marginal penalty was a constant $10 \%$ higher than average trading prices eliminated nearly all violations as expected. In this treatment, abatement cost shocks were not transmitted to emissions and violation choices, but rather were absorbed by changes in the monitoring probability.

- However, tying the marginal penalty to permit prices so that the expected marginal penalty was $10 \%$ and $50 \%$ higher than the average trading price in the Fine and High Fine treatments failed to eliminate all violations. Moreover, we observed what appear to be attempts by individuals to reduce the expected marginal penalty by selling permits at very low prices, especially in the High Fine treatment. This behavior cannot result in higher expected payoffs unless coordinated behavior results in a permit price of zero, but it does indicate the possibility that some individuals may attempt to weaken enforcement by manipulating permit prices when sanctions are based on these prices.

While the results in the Baseline and Audit treatment largely validated the underlying theory, the results from the Fine and High Fine treatments are puzzling. It is not clear why there are more violations in the Fine and High Fine treatments than in the Audit treatment. On average, the expected marginal penalties in each demand state in the Audit and Fine treatments were not different from each other, but the average expected marginal penalties were significantly higher in the High Fine treatment. Yet violations were significantly lower in the Audit treatment than the other treatments. Moreover, it is not clear to us why some individuals were motivated to try to weaken enforcement by selling permits at very low prices, even though it was not individually rational for them to do so. Future work may be able to resolve these puzzles.

It is possible that we would not observe this behavior in actual emissions markets. Sophisticated traders in high stakes environments may be less likely to engage in such costly behavior, unless they were able to successfully coordinate with other traders. Moreover, the ability of an individual or a small group of individuals to weaken enforcement with permit sales at low prices is less likely in real markets with a large number of traders. 
Still, we should not simply ignore our results because they seem unlikely in real markets. Besides, there may be simple fixes that mitigate the temptation to sell permits at low prices when these prices determine sanctions. For example, imposing a minimum penalty or basing the penalty on the median trading price instead of the average price should eliminate the influence of permit sales at low prices on the resulting enforcement strategy. 


\section{References}

Burtraw, Dallas, Karen Palmer, and Danny Kahn. 2010. A symmetric safety valve. Energy Policy 38(9), 4921-4932.

Caffera, Marcelo, and Carlos A. Chávez. 2011. The cost-effective choice of policy instruments to cap aggregate emissions with costly enforcement. Environmental and Resource Economics 50(4), 531-557.

Cason, Timothy N. and Lata Gangadharan. 2006. Emissions variability in tradable permit markets with imperfect enforcement and banking. Journal of Economic Behavior and Organization 61(2), 199-216.

Chavez, Carlos A. and John K. Stranlund. 2003. Enforcing transferable permit systems in the presence of market power. Environmental and Resource Economics. 25(1), 65-78.

Fell, Harrison and Richard Morgenstern. 2010. Alternative approaches to cost containment in a cap-and-trade system. Environmental and Resource Economics 47(2), 275-297.

Grull, Georg and Luca Taschini. 2011. Cap-and trade properties under different hybrid scheme designs. Journal of Environmental Economics and Management 61(1), 107-118.

Hood, Christina. 2010. Reviewing existing and proposed emissions trading systems. Paris: International Energy Agency.

Innes, Robert. 2003. Stochastic pollution, costly sanctions, and optimality of emission permit banking. Journal of Environmental Economics and Management. 45(3), 546-68.

Malik, Arun S. 1990. Markets for pollution control when firms are noncompliant. Journal of Environmental Economics and Management 18, 97-106.

Malik, Arun S. 2002. Further results on permit markets with market power and cheating, Journal of Environmental Economics and Management. 44(3), 371-390.

Montero, Juan-Pablo. 2002. Prices versus quantities with incomplete enforcement. Journal of Public Economics 85, 435-454.

Murphy, James J. and John K. Stranlund. 2006. Direct and indirect effects of enforcing emissions trading programs: an experimental analysis. Journal of Economic Behavior and Organization 61(2), 217-233.

Murphy, James J. and John K. Stranlund. 2007. A laboratory investigation of compliance behavior under tradable emissions rights: implications for targeted enforcement. Journal of Environmental Economics and Management 53(2), 196-212.

Newell, Richard G., William A. Pizer, and Daniel Raimi. 2013. Carbon markets 15 years after Kyoto: lessons learned, new challenges. Journal of Economic Perspectives. 27(1), 123-146.

Raymond, Leigh and Timothy Cason. 2011. Can affirmative motivations improve compliance in emissions trading programs? Policy Studies Journal 39(4), 659-678.

Roberts, M.J. and M. Spence. 1976. Effluent charges and licenses under uncertainty, Journal of Public Economics 5(3-4), 193-208.

Stranlund, John K. and Kathy K. Dhanda. 1999. Endogenous monitoring and enforcement of a transferable emissions permit system. Journal of Environmental Economics and Management 38(3), 267-282.

Stranlund, John K. 2007. The regulatory choice of noncompliance in emissions trading programs. Environmental and Resource Economics. 38(1), 99-117.

Stranlund, John K. 2008. Risk aversion and compliance in markets for pollution control. Journal of Environmental Management 88(2), 203-210. 
Stranlund, John K.; James J. Murphy, and John M. Spraggon. 2011. An experimental analysis of compliance in dynamic emissions markets. Journal of Environmental Economics and Management 62(3), 414-429.

Stranlund, John K., James J. Murphy and John M. Spraggon. 2013. Imperfect enforcement of emissions trading and industry welfare: a laboratory investigation. In Handbook on Experimental Economics and the Environment, ed. John List and Michael Price. 265-288. Northampton MA: Edward Elgar Publishing.

Stranlund, John K., and L. Joe Moffitt. 2014. Enforcement and price controls in emissions trading. Journal of Environmental Economics and Management 67(1), 20-38.

Stranlund, John K. 2017. The economics of enforcing emissions markets: a review of the literature. Review of Environmental Economics and Policy 11(2), 227-246.

U.S. Congress. 2009. American clean energy and security act of 2009. H.R. 2454, 111th Congress.

U.S. Environmental Protection Agency. 2003. Section-by-section summary of the Clear Skies Act of 2003. Washington DC U.S. EPA Office of Air and Radiation.

van Egteren, Henry and Mariane Weber. 1996. Marketable permits, market power, and cheating. Journal of Environmental Economics and Management. 30(2), 161-173.

Weber, Thomas A., and Karsten Neuhoff. 2010. Carbon markets and technological innovation. Journal of Environmental Economics and Management 60(2), 115-132.

Weitzman, Martin. 1974. Prices vs. quantities. Review of Economic Studies 41(4), 477-91.

Zhang, Zhong Xiang. 2015. Carbon emissions trading in China: the evolution from pilots to a nationwide scheme. Climate Policy. 15(sup1), S104-S126. 